\title{
Switchable Electrohydrodynamic Capillary Bridges
}

Tianxing Ma ${ }^{1}$, Darrel Dsouza ${ }^{1}$, Krysten M. Ryerson ${ }^{1}$, Matthew Signorelli ${ }^{1}$, Michael

Loewenberg ${ }^{2}$, Chinedum Osuji ${ }^{3} \&$ Jonathan P. Singer ${ }^{1}$ *

${ }^{1}$ Mechanical and Aerospace Engineering, Rutgers, the State University of New Jersey, 98 Brett Road, Piscataway, NJ 08854, USA

${ }^{2}$ Department of Chemical and Environmental Engineering, Yale University, 17 Hillhouse Avenue, New Haven, CT, 06520, USA

${ }^{3}$ Department of Chemical and Biomolecular Engineering, University of Pennsylvania, $200 \mathrm{~S}$ 33rd Street, Philadelphia, PA 19104, USA

The deformation of sessile droplets and capillary bridging in a parallel-plate capacitor under DC fields has been the subject of several scientific studies. Coaxially located droplets on opposing electrodes experience an attraction in the presence of an electric field. Application of a suitably large field will lead to either the droplets forming a liquid bridge or oscillation between bridged and de-bridged (i.e. droplet) states. We explored the bridging behavior of a variety of liquids in air. Among the liquids and droplet geometries that could form a stable field-induced bridge, only a limited set could reversibly make and break the capillary bridge by switching the electric field on and off. The ability to form a switchable liquid bridge is a function of both the liquid's 
properties, including surface tension, electric conductivity, and dielectric constant, and external conditions such as electrode separation, droplet volume.

\section{INTRODUCTION}

Liquid jets, capillary bridges, and the instability of slender liquid threads are topics that have been studied by the fluid mechanics community for centuries ever since Da Vinci's work hypothesizing that the drop's detachment from a tap is determined by the gravity overcoming the cohesive forces. ${ }^{1,2}$ The stability of a liquid bridge in the presence of an electric field has also been studied for decades. Multiple researchers have conducted studies on the stability analysis of liquid bridges of perfect dielectric ${ }^{3-6}$ and perfectly conductive ${ }^{7,8}$ fluid with $^{9}$ and without ${ }^{5,6}$ the presence of gravity. In most of these studies, however, the contact lines are typically pinned on both ends for the ease of analysis. Nonetheless, these analyses have indicated the presence of such a field could help stabilize the capillary bridge against perturbations.

Petkov et al. ${ }^{10,11}$ analyzed the capillary bridge's stability without electric field with unpinned contact lines between parallel substrates. Their study clearly shows for liquid bridges with close to $90^{\circ}$ contact angle can achieve larger aspect ratios (i.e. bridge height vs. radius) than those with contact angles that are larger or smaller.

Mugele et al. ${ }^{7}$ performed a detailed simulation analysis of the capillary bridge in an axial AC electric field using liquids with unpinned contact lines that are assumed to be perfectly conducting. In this work, the liquids are cast on the opposing surfaces of a parallel plate capacitor with the substrates coated with thin dielectric layers. By blending water, glycerol, and sodium chloride, a contact angle of about $90^{\circ}$ is achieved. Starting from this point, the electric field was applied and the droplets attracted one another to form a bridge when the voltage was high enough. The contact angle of the capillary bridge state follows the Lippmann equation ${ }^{12}$ in electrowetting on dielectric 
(EWOD): $\cos \theta=\cos \theta_{y}+\frac{\varepsilon_{0} \varepsilon_{r} U^{2}}{2 \gamma_{l v} h_{r}}$, with $\theta, \theta_{y}, \varepsilon_{0}, \varepsilon_{r}, \gamma_{l v}, h_{r}$ being the contact angle in the electric field, contact angle without electric field (Young's contact angle), the permittivity of free space, the dielectric constant of the insulation layer between the liquid and the conductive substrate, liquid-air interfacial energy, and the thickness of the insulation layer respectively. In contrast to the bridge state, the droplets take the Young's contact angle without any contribution from the electric field. While the voltage is smaller than the critical voltage, an effective "phase diagram" can be composed of three regimes: droplets at large separation, capillary bridges at small separation, and a coexistence area at intermediate gap size, of which the intermediate state's morphology is history-determined (i.e., if initially the liquid is in droplets state, they will stay as droplets after the electric field is turned on and vice versa). Above a critical voltage, oscillation replaces the coexistence area and represents an unstable behavior of periodic oscillation between droplets and capillary bridges at a constant electric field. The authors attributed the oscillation phenomena from the electrowetting during the bridge state: the contact angle remains relatively constant during the bridge state, but the wetting diameters get larger, which drives the bridge unstable and breaks it back into droplets. The droplets then repeat the initial process, attracting each other and then form the capillary bridge. The frequency of the oscillation is determined by the kinetics of the charge relaxation process through the insulation layers. The breaking of the liquid bridge in the oscillation process is claimed to be an electrowetting process with advancing contact line and a constant contact angle.

Mugele et al.'s ${ }^{7}$ work has clearly shown the possibility to use a simple configuration to achieve the actuation of droplets without any motion of the substrates. However, for the EHD capillary bridge to serve as an actuator fully controlled by the switching of the electric field, the behavior of the liquid after the electric field turning off needs to be analyzed to make sure the capillary bridge 
formed could be broken back into droplets. Thus, to develop a motion-on-demand-only capillary bridge switch, it is of interest to investigate the whole process of the EHD capillary bridge switch, i.e., the formation of a stabilized capillary bridge by turning on the electric field and the breaking of the capillary bridge back into droplets by switching off the electric field. Further, it would be desirable to accomplish this using non-volatile liquids to create a device that could function indefinitely.

In this work, we demonstrate that by using different liquids, gap sizes, and voltages, droplets with volumes $<15 \mu \mathrm{L}$ placed in a parallel plate capacitor, different modes of motion can be achieved by changing the DC electric field. Most interestingly, we found a mode of motion which enable the droplets to form a capillary bridge when the electric field is turned on and break back into droplets states when the electric field is switched off, but only for certain fluids. This repeatable bridge-on-demand behavior using such an simple configuration makes it possible for applications like scalable manufacture of laminar device for applications in, for example, thermal switches. ${ }^{13,14}$ 


\subsection{Experimental}

a

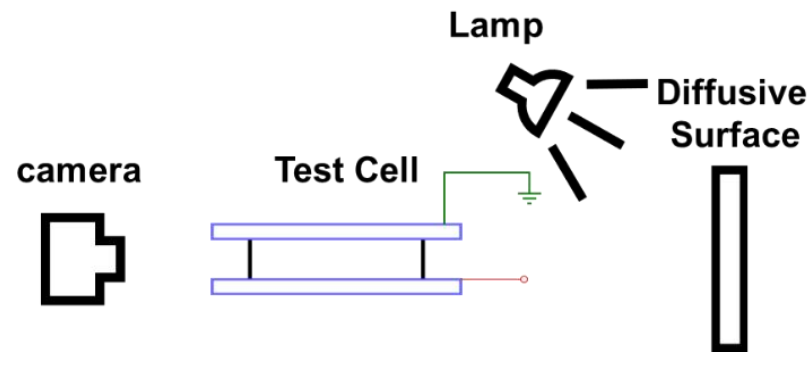

b

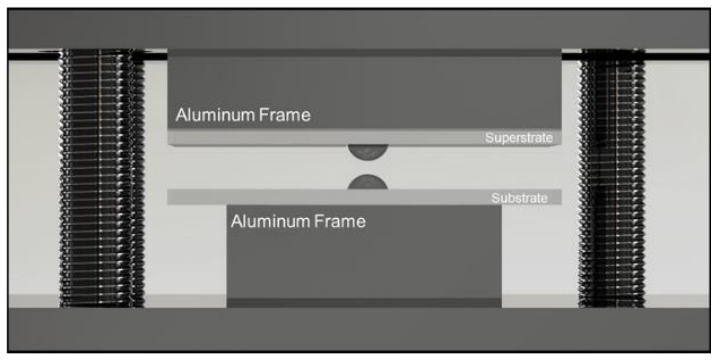

Figure 1. (a) Schematic drawing of the experimental set up of the switchable EHD capillary bridge: The top and bottom aluminum frames are grounded; the top surface of the bottom substrate is connected to a high voltage power supply. (b) Schematic drawing of the test cell. The threaded posts are the fine adjustment screws used for gap control.

Two pieces of $25.4 \mathrm{~mm} \times 25.4 \mathrm{~mm} \times 1 \mathrm{~mm}$ ITO glass are used as the substrates for the EHD tests. For both substrates, the ITO surface faces upward where the top ITO is grounded while the bottom ITO is connected to a positive polarity high voltage DC power supply (Acopian P012HA2.5). The gap size is controlled by 3 fine adjustment screws. The voltage of the high voltage power supply is controlled by a custom LABVIEW code. A CMOS camera is used for measuring the gap size and recording the motion of the liquid with a frame rate of $25 \mathrm{fps}$. Light from a lamp is projected to a scattering back surface for illumination.

The droplet is cast from a micropipette onto the bottom substrate after a thorough cleaning of the substrates using an excess amount of acetone and isopropyl alcohol. The top substrate, hereinafter referred as the superstrate, is brought down to contact the droplet and raised to break the capillary bridge formed to set the initial condition of two opposing droplets. Unless specifically stated, the voltage is turned on from $0 \mathrm{~V}$ to the set value in a step function manner and shut off in 
the same method. The surfaces of the ITOs are modified by using self-assembled monolayers (Gelest (tridecafluoro-1,1,2,2-tetrahydrooctyl)silane) to modify the contact angles and minimize contact angle hysteresis. Since these coatings are affected by the heating and cleaning over the course of experiments, the contact angle was also extracted from the droplet videos to capture the current state of the surface.

\subsection{Simulation}

The frames of the test videos are processed by a custom MATLAB image processing code to extract the edge of the liquid profile. The extracted profiles are then exported into COMSOL Multiphysics for the calculation of the free energy using the steady-state electric current model. Both the image processing and the free energy calculation are described in detail in the Supporting Information.

2.3 Materials

All the EHD fluids are commercially available products. The canola oil, N-methyl-1,2pyrolidone (NMP), hexadecane, terpineol, castor oil, glycerol trioleate, polyethylene glycol (average Mn 400) are used as purchased from Millipore Sigma. 1-Ethyl-3-methylimidazolium tetrafluoroborate, $>98 \%$ (IL-0006-HP-0100) is used as purchased from Iolitec. The glycerol is used as purchased from PTI Process Chemicals. The dielectric oil is used as purchased from Miles Lubricants (Voltage II, Inhibited Electrical Insulating Oil). Glycerol triacetate is used as purchased from Alfa Aesar. Glycerol tricaprylate is used as purchased from Stepan (Neobee 895). Silicone oil (350 cSt) is used as purchased from Consolidated Chemical \& Solvents LLC. 


\section{RESULTS}

\subsection{Phase Diagrams}

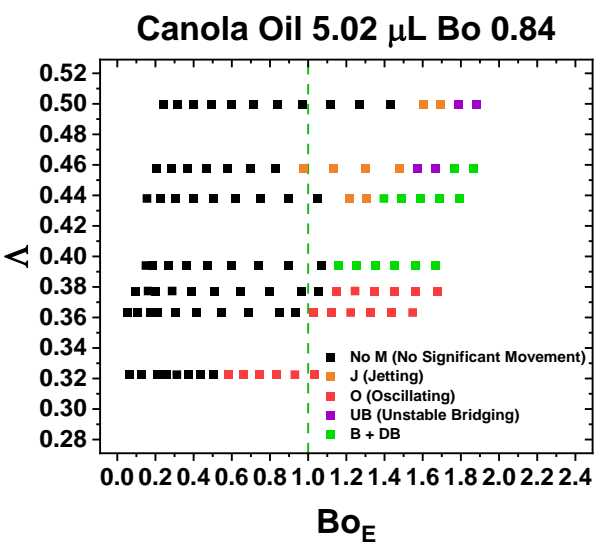

Figure 2. Phase diagram of $5 \mu \mathrm{L}$ canola oil. The $x$-axis is the electrical Bond number in the air gap using the electric field strength in the air domain without any influence from the liquid. The $y$-axis is the slenderness ratio. The reference line is a guidance indicating where the critical bulk $B o_{E}$ is 1.

By adjusting the voltage and gap size between the substrate and the superstrate, behaviors of specific volumes are plotted onto phase diagrams. The electrical bond number (also referred to as electrocapillary number) here is defined as $B o_{E}=\frac{\varepsilon_{0} a E^{2}}{\gamma}$, characterizing the relative importance of electric force versus the capillary force, where $\varepsilon_{0}$ is the vacuum permittivity. The characteristic length $a$ takes the value of $a=V^{1 / 3}$ and the strength of the electrical field, $E$, is approximated by the strength of the electrical field in the air gap. The surface tension of different liquids from literature are used where available (Table S1). The slenderness on the $y$-axis is defined by $\Lambda=$ $H / 2 a$, where $H$ is the gap size. This is a dimensionless number characterizing how slim the capillary bridge is. 
By observing the fluid after switching the electric field on and off, the motion of the fluid can typically be classified into five different regimes: No movement, jetting (J), successful bridging and debridging $(\mathrm{B}+\mathrm{DB})$, oscillating $(\mathrm{O})$, and bridging only $(\mathrm{BO})$. The different behaviors are described as follows:

\subsection{Bridging and Debridging process:}

3.2 a) Bridging process
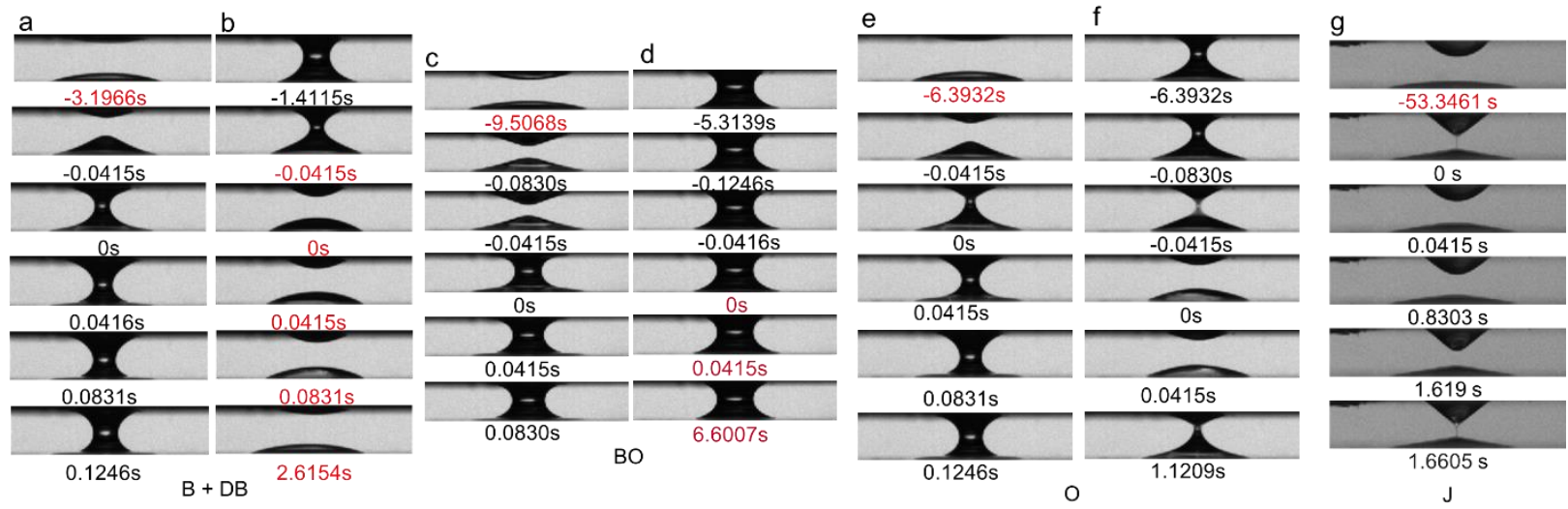

Figure 3. Side view snapshots of four characteristic behaviors of pendent droplets in electric field. $6.96 \mu \mathrm{L}$ canola oil is used for $(\mathrm{a})-(\mathrm{f})$ and $8.6 \mu \mathrm{L}$ Triton $\mathrm{X}-100$ is used in $(\mathrm{g})$. The red text indicates that the electric field is off; the black text means the electric field is on. The $0 \mathrm{~s}$ is the first frame after the liquid's morphology change (or switching off of the electric field for the BO case in (d). (a) - (b) the liquid's behavior after electric field's switching on and off for a B + DB case where $B o_{E}=1.13, \Lambda=0.50(3 \mathrm{kV}, 1.7 \mathrm{~mm})$. (c) - (d) the liquid's behavior after the electric field's switching on and off for a $\mathrm{BO}$ case where $B o_{E}=0.45, \Lambda=0.41(1.6 \mathrm{kV}, 1.4 \mathrm{~mm})$. (e) - (f) the liquid's behavior after electric field's switching on and off for an $\mathrm{O}$ case where $B o_{E}=0.95, \Lambda=$ 
$0.50(2.75 \mathrm{kV}, 1.7 \mathrm{~mm}) .(\mathrm{g})$ the liquid's jetting behavior of $8.6 \mu \mathrm{L}$ Triton $\mathrm{X}-100$ with $B o_{E}=0.92$, $\Lambda=0.43(2.5 \mathrm{kV}, 1.8 \mathrm{~mm})$

As seen in Fig.3a, with the gap size set at $1.7 \mathrm{~mm}$, using a voltage of $3 \mathrm{kV}$, after turning on the electric field, the opposing droplets start to attract each other, moving the contact line towards the axis (Fig. 3a and Fig. S1a) and shrinking the air gap between them. With evolution of time, the instability develops and the droplet forms a Taylor cone to jet the liquid towards the opposing droplet. The jet then merges with the opposing droplet and develops into a capillary bridge connecting the substrate and superstrate.

The capillary bridge formed then thickens at the neck region. During the thickening of the bridge neck, convective flow inside the bridge redistributes the mass of the liquid: When the capillary bridge forms $(0 \mathrm{~s}$ in Fig. 1a), the liquid bridge looks symmetric with respect to the neck. In the next frame (0.0416 s in Fig. 1a), however, more mass is distributed to the top part of the liquid bridge, and the neck position is shifted downward from the center. This downward movement of the neck's location stops around $0.1246 \mathrm{~s}$ (Fig. 1a $0.1246 \mathrm{~s}$ ) after the bridging, and the neck location starts to move back at $0.16 \mathrm{~s}$ after the bridging, and this position oscillation diminishes in magnitude and becomes unrecognizable afterward. With the volume of the jet provided from the bottom droplet, a broadening of the wetting diameter on the superstrate is also seen (Fig. S1 a). The change in neck width then slows until the electric field is turned off.

After the bridge forms, the wetting diameter on the substrate continues shrinking by up to $26.5 \%$ compared with the wetting diameter at $0.0831 \mathrm{~s}$ after the bridging. Another phenomenon worth noticing is the appearance of a thin layer of liquid close to the substrate: this thin layer of liquid deforms together with the bulk liquid initially; however, with the evolution of time, the deformation of this layer lags behind the bulk's and becomes apparent at $0.04 \mathrm{~s}$. This deformation 
is slow but does not halt. At $0.0831 \mathrm{~s}$ after bridging, this layer becomes indistinguishable to the camera. This layer's slow motion might be a result of its small curvature, as the flow in the system is driven primarily by the surface tension and the viscous stress near the substrate.

During the bridging process, the apparent contact angle oscillates while the droplets approach each other (Fig. S1c). The contact angle on the bottom substrate quickly decreases after the capillary bridge's formation as the liquid is pulled into the bridge. After the thin layer disappears, the liquid bridge shows a higher contact angle than the droplets.

As a summary, in the $\mathrm{B}+\mathrm{DB}$ (bridging with debridging) regime with the field on, the liquid forms a capillary bridge with a smaller footprint on the substrates and a higher contact angle with the presence of the electric field.

\section{2 b) Debridging Process}

When the electric field is turned off, the liquid bridge's neck thins and breaks back into two droplets (Fig. 3b). The droplets then slowly wet the superstrate and substrate, relaxing back into the original two droplets morphology. Comparing with the switching on process, the relaxation of the liquid takes a longer time (Fig. S1b,d).

\subsection{Description of Other Regimes:}

The $\mathrm{B}+\mathrm{DB}$ regime is not always guaranteed at all experimental conditions. The behavior of the droplets/capillary bridges is influenced by parameters including but not limited to volume, electric field, gap size, contact angle, and liquid properties such as surface tension, density, dielectric constant and electrical conductivity. 
As a result, there are other characteristic behaviors. Of all the liquid tested in this manuscript, the characteristic behaviors are summarized into five regimes based on their observed motion mode. Other than the $\mathrm{B}+\mathrm{DB}$ regime described in the prior section, there are four other regimes observed : (1) "No significant movement (No M)": No capillary bridge formation could be captured by the camera; (2) "Jetting (J)": The droplets could jet liquid into the opposing substrate, but this behavior could not induce a capillary bridge; (3) "Bridging only (BO)": Capillary bridge could form by switching on the electric field, but the liquid bridge could not break back into droplets by turning off the electric field; (4) "Oscillating (O)": The liquid bridge-droplets morphology change happens in cycles while the electric field is turned on and kept at a constant voltage.

\section{3 a) Bridging Only}

At a small slenderness (i.e., gap size), if the $B o_{E}$ is large enough to initiate a capillary bridge, the two droplets might coalesce and form a capillary bridge between the substrate and the superstrate as described previously. When the electric field is turned off, the capillary bridge remains the bridge state without breaking into droplets. We call this type of behavior "Bridging Only (BO)". The bridging process is similar to the B + DB regime. When the electric field is turned off, a thinning of the bridge neck and an increase in the contact angle can be observed.

\section{3 b) Oscillating $(\mathrm{O})$}

Using the same gap size and a lower voltage (e.g., $1.7 \mathrm{~mm}, 2.75 \mathrm{kV}$ for a $6.96 \mu \mathrm{L}$ droplet of canola oil) as the B + DB case, after turning on the electric field, the droplets show the same behavior as the $\mathrm{B}+\mathrm{DB}$. However, after the bridging, although the voltage is still on, an instability 
develops and the capillary bridge breaks back into droplets and then repeat the process of bridging and debridging. If the electric field is not modified, this oscillation will continue.

Comparing Fig. 3a and Fig. 3e we can find the neck diameter formed in this case after the stabilization of the first bridging is smaller than the $\mathrm{B}+\mathrm{DB}$ case. With the same surface tension and gravitational force, the larger electric forces from the higher electric field stabilized the capillary bridge. $^{5}$

\section{3 c) Jetting}

Under specific experimental settings, a thin thread of liquid will jet from the Taylor cone formed from one droplet towards the opposing droplet, similar with the initial stage of the bridging process. However, in contrast with the $\mathrm{O}$ regime, the formation of a jet between the two droplets does not lead to a liquid bridge and the thread typically last much shorter time than the oscillation. This phenomenon is called cone jet in other EHD applications such as electrospray. ${ }^{15}$ Under extended observation of the droplets, we can observe the J regime (Fig. 3g), where thin thread of liquid could be observed. Although a frame rate of 25 fps does not always capture the jetting behavior, the relaxation of the droplet after jetting a liquid can typically be observed. In the phase diagrams, this behavior typically exists at a higher $B o_{E}$ than the No M points, but before transit into the $\mathrm{O} / \mathrm{B}+\mathrm{DB}$ regime. Geometrically, a thinner jet results in a larger curvature, making it

more likely to break before reaching the opposing droplet, preventing the formation of a continuous interface and the subsequent evolution after the formation of a neck. Simultaneously, a thin thread liquid jet could develop a sharp tip. For droplets approaching each other, even if they carry opposite charges, sharp tips are considered an unfavorable geometry for coalescence and 
thus causes quick repulsion of the opposing droplets. ${ }^{16,17}$ Consequently, J behaviors will also be expected to take a larger area of the phase diagram when the slenderness is of larger value.

\subsection{Behavior of Other Materials}

Different liquids possessing a wide range of properties have been tested using this method to determine if the $\mathrm{B}+\mathrm{DB}$ regime can be achieved. The related properties are listed in Table $\mathrm{S} 1$ and the derived characteristic values are listed in Table 1 in section 4.6. Two types of silicone oil with different viscosity are picked for their low electrical conductivity and surface tension. NMP is picked for its high dielectric constant. Glycerol is selected due to its high dielectric constant and high surface tension. Hexadecane is used for its slightly higher conductivity. Polyethylene glycol has lower electrical conductivity and higher dielectric constant. [EMIM] $\left[\mathrm{BF}_{4}\right]$ is an ionic liquid that has a high electrical conductivity compared with all other materials. Castor oil is selected for its higher viscosity and lower electrical conductivity. A dielectric oil (light naphthenic petroleum distillate) is a lower electrical conductivity material with a lower dielectric constant. As seen in Fig. 4, most liquids, except for castor oil, do not have a B+DB state on their phase diagrams. 

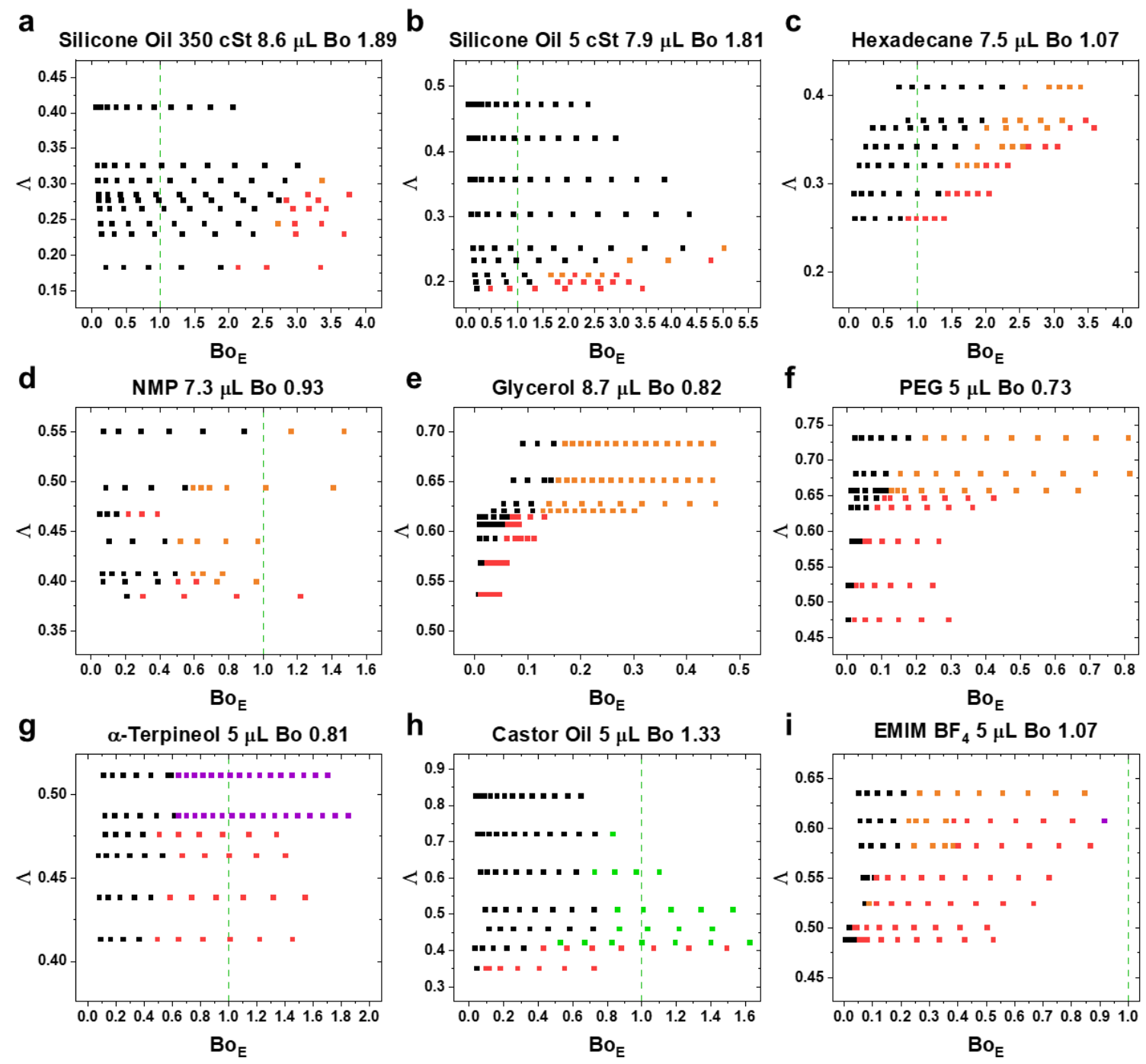

Figure 4. Phase diagram for different material with $B o_{E}$ being the electrical Bond number and $\Lambda$ being the slenderness. (a) $8.6 \mu \mathrm{L} 350 \mathrm{cSt}$ silicone oil (b) $7.9 \mu \mathrm{L} 5 \mathrm{cSt}$ silicone oil (c) $7.5 \mu \mathrm{L}$ hexadecane (d) $7.3 \mu \mathrm{L}$ NMP (e) $8.7 \mu \mathrm{L}$ glycerol (f) $5 \mu \mathrm{L}$ PEG (g) $5 \mu \mathrm{L} \alpha$-terpineol (h) $5 \mu \mathrm{L}$ castor oil (i) $5 \mu \mathrm{L}$ EMIM BF 4

Additional tests were carried out on liquids with similar molecular structure as canola oil (Fig. 5): Glyceryl triacetate(C 1:0/1:0/1:0 MW $218.2 \mathrm{~g} / \mathrm{mol})$, glyceryl tricaprylate (C 8:0/8:0/8:0 MW $470.7 \mathrm{~g} / \mathrm{mol}$ ) and glyceryl trioleate $(\mathrm{C}$ 18:1/18:1/18:1 $\mathrm{MW} 885.4 \mathrm{~g} / \mathrm{mol})$. For the short chain triglyceride(glyceryl triacetate), as its properties are quite different from the ones of canola oil, a 
different phase diagram shows up as expected: the bridging behavior could be achieved at a lower $B o_{E}$ but no $\mathrm{B}+\mathrm{DB}$ is observed and when the electric field is high, the liquid shows the $\mathrm{O}$ state instead. With longer chain length, the glycerol tricaprylate starts to show an area of B+DB. Using an even longer chain length molecule requires a higher $B o_{E}$ to generate motion of the droplet under the same slenderness, but enables the $\mathrm{B}+\mathrm{DB}$ behavior at a lower slenderness.
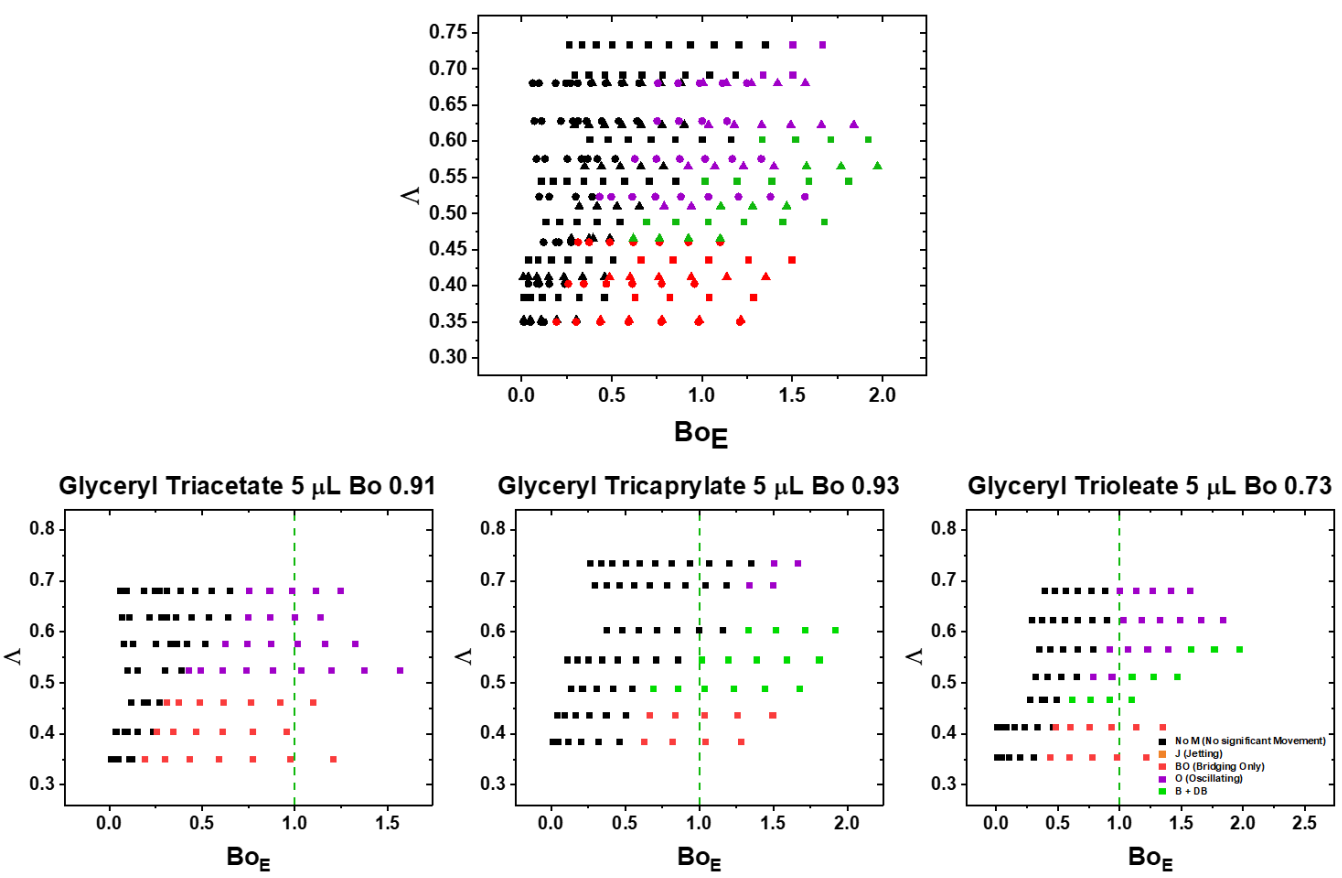

Figure 5. Phase Diagram of Different Triglyceride, with circles showing the behavior of glyceryl triacetate, squares showing glycerol tricaprylate, and triangles showing the glyceryl trioleate.

\section{DISCUSSION:}

Based on the behavior of liquid after the electric field turns on, the mechanism of the switchable capillary bridge in electric field can be broken down into several well-studied processes: (1) tip streaming of pendant charged droplets; (2) coalescence of charged jets/droplets; (3) stabilization 
of capillary bridge in electric field; and (4) the capillary bridge's stability. An analysis each is presented in this section and the results are used to generate a $B o_{E}-\Lambda$ phase diagram for different liquids.

\subsection{Tip Streaming Process of Charged Droplets}

Tip streaming has been a main subject of EHD studies for over a century. ${ }^{18}$ It describes the behavior of a droplet developing a Taylor cone at the presence of electric field and forming a cusp at the tip to shoot a thread of liquid from the Taylor cone. Two assumptions are generally accepted for this process: (1) The liquid's bulk geometry, whether the liquid is a film ${ }^{21}$, a pendant droplet pinned on a substrate ${ }^{22}$, a pendant droplet at the outlet of a capillary tube ${ }^{19}$, or a floating droplet $t^{23}$, ${ }^{24}$, is considered not to play any important role for the tip streaming process. Instead, the characteristic field strength is typically set by the local geometry of the jet developed like the radius of the jet. (2) The tip streaming happens at a narrow range of electric field thus the electric field strength plays little role once this range has been reached. Balance of electrical stress with surface tension to initiate a jet, i.e. $B o_{E}=\frac{\varepsilon_{0} d_{0} E_{0}^{2}}{\gamma} \sim 1$, where the characteristic length during the tip streaming process $d_{0}$ is set by $d_{0}=\left(\frac{\varepsilon_{0}^{2} \gamma}{\rho K^{2}}\right)^{1 / 3}$ based on the fact the most of the kinetic energy of the tip streaming process comes from the electrical energy. ${ }^{19,20,24} E_{0}, \gamma, \rho$ and $K$ are the electric field strength outside the fluid, surface tension, density, and electrical conductivity of the liquid, respectively. The characteristic time scale for the tip streaming is the capillary time $t_{c}=\sqrt{\frac{\rho d_{0}^{3}}{\gamma}}$, which is the same order of magnitude as the characteristic charge relaxation time $t_{e}=\frac{\varepsilon_{0}}{K}{ }^{22}$ In a recent work, Gañán-Calvo et.al ${ }^{19}$ derived the scaling law for the first droplet emission process of 
electrospray. From their analysis, we derive the effective kinetic energy $F_{k}$ of the jetting process assuming the geometry of the jet as a column with radius $R_{0}$ and length of $L_{0}$ :

$$
F_{k} \sim \pi \rho R_{0}^{2} L_{0} v_{z}^{2}
$$

, where $v_{z}=\varepsilon_{r}^{-\frac{1}{4}} v_{0}, L_{0}=\delta_{\mu}^{-1} \varepsilon_{r}^{\frac{1}{4}} d_{0}, R_{0}=\varepsilon_{r}^{\frac{1}{2}} d_{0}$, and $\delta_{\mu}=\frac{\rho d_{0} v_{0}}{\mu}$ are the corresponding end speed of the tip streaming, length, and the front radius of the jet and a dimensionless number similar to Reynolds number following the definition from Gañán-Calvo et.al ${ }^{19}$.

\subsection{Coalescence of Charged Droplets}

When droplets with opposite charges approaching each other, the coalescence of them is not always guaranteed. ${ }^{16,17}$ The coalescence is determined by the local geometry of the neck formed between the two charged cones. Bird et. al, found that critical half angle of the neck region needs to be larger than $59.2^{\circ} .{ }^{16}$

Assuming a case where two fully developed jets meet each other, the neck half angle can be approximated by

$$
\theta_{0} \sim \operatorname{atan}\left(\frac{R_{0}}{L_{0}}\right)=\operatorname{atan}\left(\delta_{\mu} \varepsilon_{r}^{\frac{1}{4}}\right)
$$

\subsection{Axial electric field stabilized capillary bridge}

It has been demonstrated that the presence of an axial electric field could greatly enhance the stability of a liquid bridge when the condition $\frac{K}{K_{0}}-\frac{\varepsilon}{\varepsilon_{0}}>0$ is met . ${ }^{5,25,26}$ As air typically has a low electrical conductivity $\left(<1 \times 10^{-14} \mathrm{~S} / \mathrm{m} \text { at ambient temperature }\right)^{27}$, this condition is generally satisfied. The higher the $B o_{E}$, the larger the critical slenderness the bridge could achieve. ${ }^{28}$ As seen 
in Fig. 3, increasing the electric field could stabilize a bridge from an $\mathrm{O}$ state to $\mathrm{B}+\mathrm{DB}$ state. However, the introduction of gravity also influence the stability to a large extent, ${ }^{5}$ the slenderness achieved in our work for the bridging cases are relatively small compared with those tests conducted with lower $B 0^{5,25,26}$ 
4.4 Geometric Description and Free Energy Understanding of the Bridging and Debridging Processes

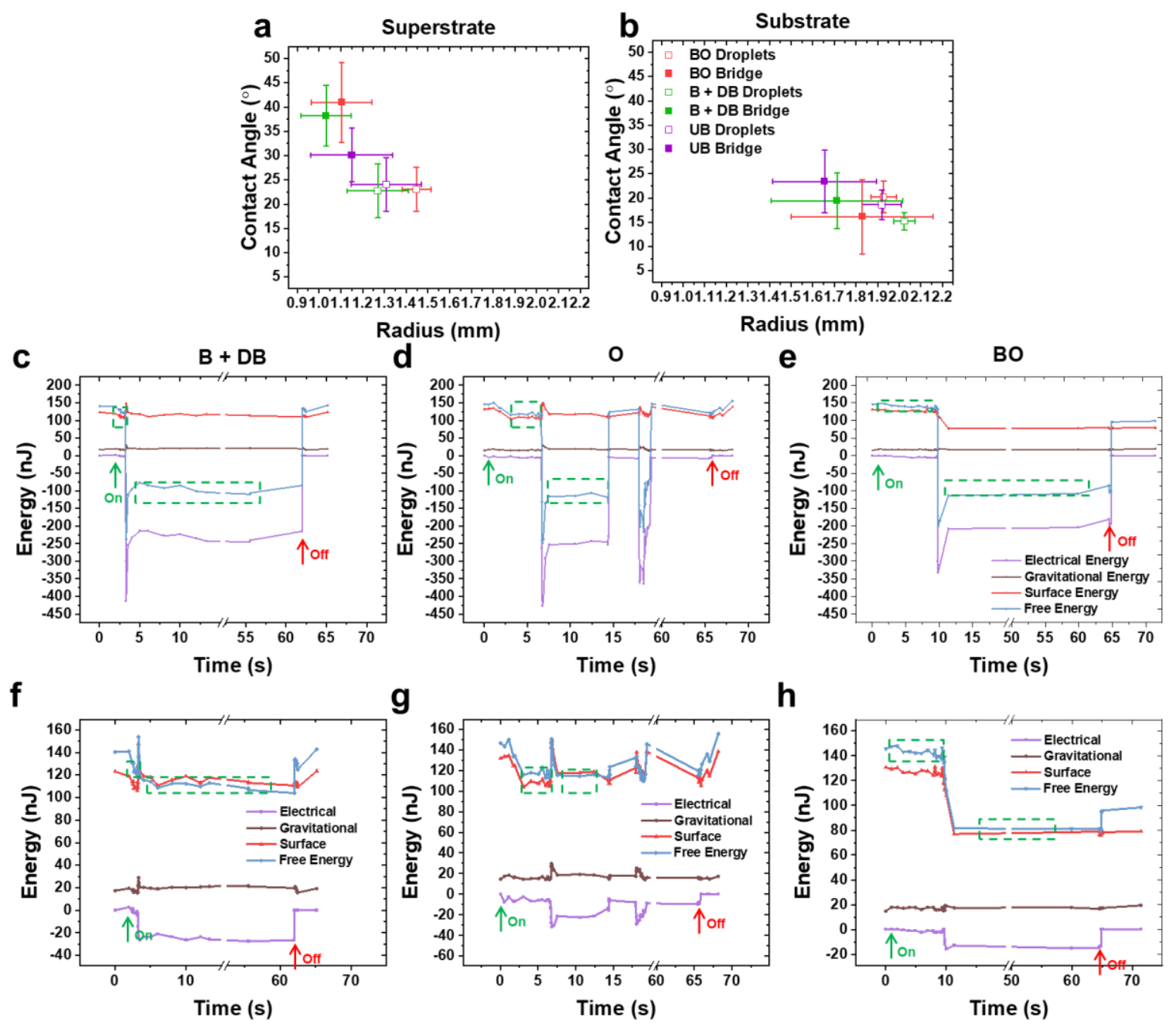

Figure 6. Contact angle versus radius of $6.96 \mu \mathrm{L}$ canola oil for the $\mathrm{BO}, \mathrm{B}+\mathrm{DB}$, and $\mathrm{O}$ states on the (a) superstrate (b) substrate. Free energy plots for the $6.96 \mu \mathrm{L}$ canola oil for the three states assuming charge relaxation has completed: (c) B+DB, (d) O, and (e) BO. Free energy plots for the $6.96 \mu \mathrm{L}$ canola oil for the three states assuming charge relaxation has not started: (f) $\mathrm{B}+\mathrm{DB},(\mathrm{g})$ $\mathrm{O}$, and (h) BO. The electrical energy is offset by subtracting the electrical energy by the initial electrical energy of the system. 
During the bridge state, the contact radius is smaller than the droplets state, and the contact angle becomes larger. This again confirms our case is different from the typical electrowetting phenomena as has been described by Klingner et al. ${ }^{7}$ The free energy of the three states is also plotted (Fig. 6 c-h). The free energy is approximated by the summation of electrical energy, gravitational energy, and the surface energy as described in the method section and supporting information. The electrical energy calculated here is based on the two assumptions: $1 . t_{e} \ll t_{c}$ for Fig. $6 \mathrm{c}$-e where the circuit has reached a steady state (i.e., the charge decay process has completed as the charge relaxation time is much shorter than the capillary time). 2. $t_{e} \gg t_{c}$ for Fig. $7 \mathrm{f}-\mathrm{h}$ where the charge decay is much slower than the characteristic experimental time. As the charge relaxation is slower than the fluid flow during the tip streaming process, the electrostatic simulation (Fig. $6 \mathrm{f}$-h) can be expected to be closer to the real case during the tip streaming and coalescence process. Concurrently, the electric current simulation is expected to be closer to the steady state when the profile is not changing much. The electrical energy is derived from the COMSOLsimulated difference between the frame analyzed and when the electric field is first turned, which may lead to some minor distortion from the difference in droplet shape, but effectively removes the rest of the simulation cell from consideration. The charge relaxation process during the experimental process should be a middle state between the two simulated extremes. The kinetic energy is not represented on these plots as in most cases the geometry evolution is small between frames.

Although the kinetic energy is neglected, we can get information from the free energy of the "stable states" in electric field (green rectangles in Fig. 6 c-e). These stable states are where the profile has evolved after a while after the field is turned on and when the profile is not changing 
much over time. To avoid the BO case, the surface energy of the bridge state needs to be larger than the deformed droplets to provide the driving force to break the liquid bridge after electric field switched off. The mechanism of the $\mathrm{O}$ state is more complex. The electrostatic simulation suggests that the unstable bridge is a result of the free energy of the bridge being similar or higher than the free energy of the droplets; however, we expect that the electrostatic case is most valid during the early evolution of the droplets when thermodynamic interpretations are the least accurate. In the electrical current model, the bridges are always much more stable than the droplets. Since the O regime is, like the $\mathrm{J}$ regime, a more dynamic behavior, it is possible that some combination of charge and kinetic effects not captured by the model make the electrostatic simulation more representative in these cases. Either way, it appears as if the electrostatic simulation can effectively predict when the $\mathrm{O}$ regime will occur, and thus is at least a useful model for this analysis.

The sharp spikes of the surface energy between the droplets in field and the bridge state are from the high surface energy of the slender column and the retracting wetting layer on the substrates during the bridging process (Fig. $6 \mathrm{f}-\mathrm{h}$ ). These act as barriers to the spontaneous transition between states. Based on the dimensional analysis from Equation 1(See Tab.1), the kinetic energy of the tip streaming is on the order of $\sim 100 \mathrm{~nJ}$ assuming a jet length of $\sim 0.1 \mathrm{~mm}$, which one order of magnitude larger than the energy barrier from the surface energy. As the Reynolds number is typically $<1$, the viscous dissipation could aid in surpassing the energy barrier. The use of a higher voltage could move more liquid from quiescent to moving and thus there is more kinetic energy dissipation, which may also favor the $\mathrm{B}+\mathrm{DB}$ behavior. 
4.5 Behavior of droplets/capillary bridge in electric field: Flowchart and phase diagram
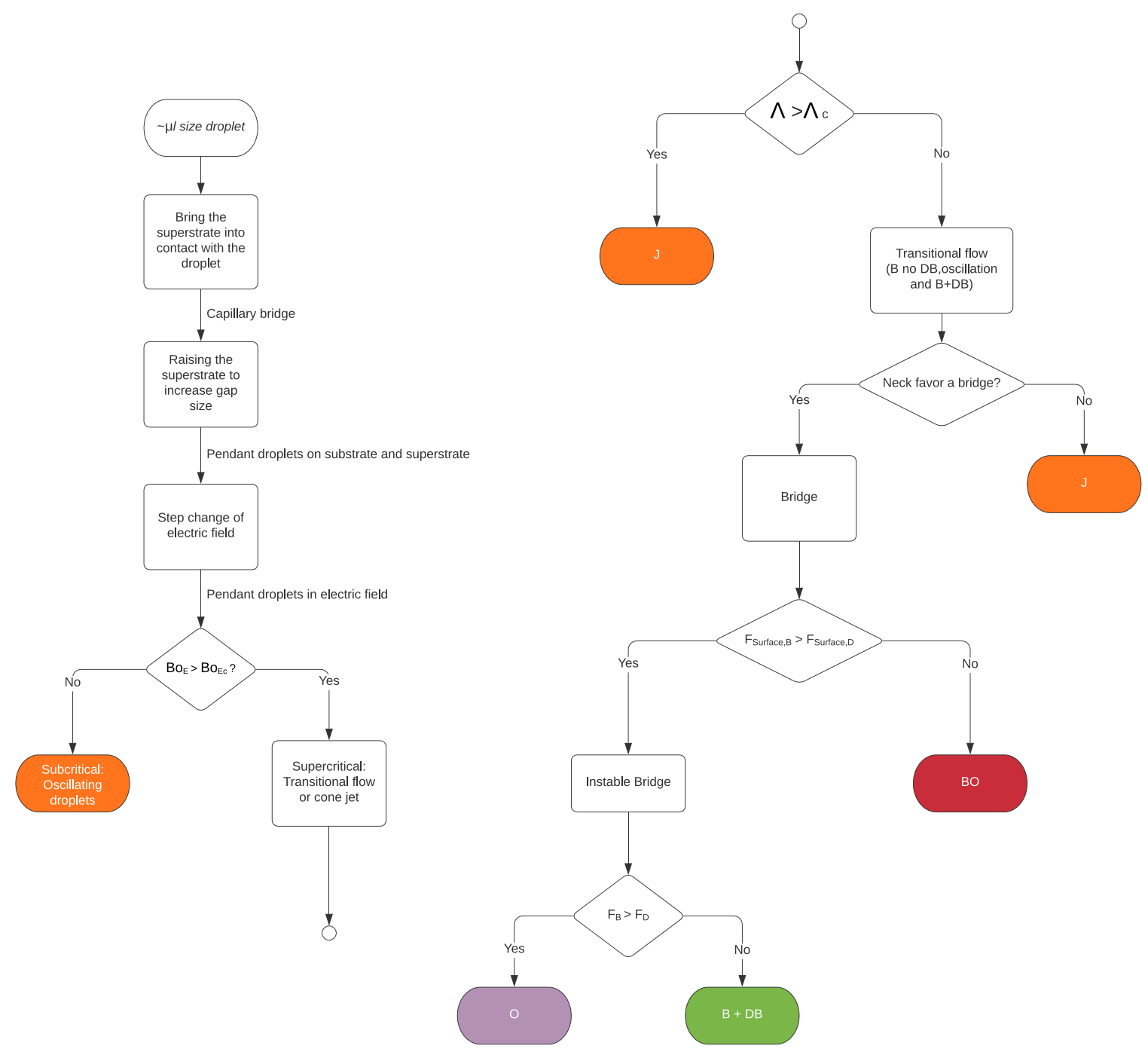

Figure 7. Flowchart of pendant droplets in DC electric field

Based on the process the liquid undergoes, the different modes of motion are summarized into a flowchart (Fig. 7). The droplets in the DC electric field is subjected to these processes:

(1) A droplet of $\sim$ ul size is cast on the substrate

(2) The superstrate is moved down to get in touch with the droplet

(3) A capillary bridge forms between the substrate and the superstrate 
(4) The superstrate is moved up to increase the gap size to break the capillary bridge into pendant droplets on the substrate and the superstrate

(5) After waiting for $>1 \mathrm{~min}$, the electric field is turned on using an electronic switch. The electric field ramps up rapidly using a step function

(6) The introduction of the EHD forces start to change the morphology of the droplets. Based on the magnitude of the $B o_{E}$ they can exhibit different behaviors.

(7) As described by Ferrera et. $\mathrm{al}^{22}$, when the $B o_{E}$ number is below a critical value, the droplet would form circular flow inside and oscillate in the axial direction by stretching the apex with decaying amplitude due to the viscous dissipation, on the contrary, while the $B o_{E}$ number is above that critical value, the droplets will form tip streaming and eventually jet out smaller droplets given enough room and time. Ganan, et. al derived that this critical $B o_{E}$ (electric field strength) based on the balance of jet inertia and surface tension. ${ }^{19,}{ }^{29}$ In our case, however, as the liquid has been split into two pendant droplets, this number could be smaller than 1. As a result, for supercritical droplets, to form a capillary bridge, the two opposing droplets not only need to get into contact with each other, but also need to make sure they can coalesce without breaking into smaller droplets through the Rayleigh instability or Coulomb fission.

(8) If the gap size is large enough, the transitional flow will be able to develop into the cone jet mode to eject droplets. As the continuous interface has already been broken after the droplet's formation, the capillary bridge could not form thus this can be viewed as the jetting mode. The tip streaming flow has been analyzed by Collins, et al. ${ }^{30}$ Their work demonstrates that fluid with longer charge decay time enables longer tip streaming before the emission of the first droplet. 
(9) If the gap size is not large enough, the transitional flow could not have enough room to incept the shedding of droplet from the jet thus keeps the continuous flow condition. To the contrary, if the gap size is too large, the tip streaming could develop and start to shed off smaller droplets at the tip of jets, then the continuous flow is broken and the jets could not merge.

(10) If the opposing jet can meet each other and coalesce, a liquid bridge forms between the pendant droplets. If not, the droplets will repel each other without forming a bridge. Thus, it will fall into the $\mathrm{J}$ regime.

(11) If the liquid bridge can form and further develops into a geometry to minimize the surface energy to a value smaller than the droplets state before the turning on of the electric field, the bridge will not be broken after turning off the electric field, thus it is in a BO regime.

(12) When this condition is not met, the bridge formed is an unstable or metastable geometry. However, with the electrical energy lowering the total free energy, the total free energy of the bridge state could be lowered thus making it a favorable geometry in the electric field. This is the desired B + DB state. When the electric field couldn't provide enough change in the free energy to make the bridge state an energy favorable state or if dynamic effects prevent reaching the thermodynamically predicted behavior, the bridge could not be stabilized and forms the $\mathrm{O}$ state.

\subsection{Comparison between Different Materials}




\begin{tabular}{|c|c|c|c|c|c|c|c|c|c|}
\hline Liquid & $\begin{array}{l}d_{0} \\
(\mathrm{~m})\end{array}$ & $\begin{array}{l}t_{c} \\
\text { (s) }\end{array}$ & $\begin{array}{c}\boldsymbol{E}_{\mathbf{0}} \\
(\mathbf{V} / \mathbf{m})\end{array}$ & $\begin{array}{c}E_{0 B} \\
(\mathbf{V} / \mathbf{m})\end{array}$ & $\begin{array}{l}L_{0} \\
(\mathbf{m})\end{array}$ & $\begin{array}{c}v_{z} \\
(\mathbf{m} / \mathbf{s})\end{array}$ & $\begin{array}{l}R_{0} \\
(\mathbf{m})\end{array}$ & $\begin{array}{l}\boldsymbol{F}_{\boldsymbol{k} 0} \\
(\mathbf{J})\end{array}$ & $\begin{array}{l}\theta_{0} \\
\left({ }^{\circ}\right)\end{array}$ \\
\hline Silicone Oil - 5 cSt & $5.52 \mathrm{E}-01$ & $8.85 E+01$ & $6.35 E+04$ & $1.14 \mathrm{E}+06$ & $1.02 \mathrm{E}-03$ & $4.91 \mathrm{E}-03$ & $8.90 \mathrm{E}-01$ & $5.51 \mathrm{E}-05$ & 89.90 \\
\hline Silicone Oil - 350 cSt & $5.54 \mathrm{E}-01$ & $8.85 E+01$ & $6.56 E+04$ & $1.18 \mathrm{E}+06$ & $7.20 \mathrm{E}-02$ & $4.86 \mathrm{E}-03$ & $9.19 \mathrm{E}-01$ & $6.09 \mathrm{E}-05$ & 89.94 \\
\hline $\begin{array}{c}\text { N-Methyl-2- } \\
\text { Pyrrolidone (NMP) }\end{array}$ & $1.98 \mathrm{E}-04$ & 4.43E-04 & $4.83 \mathrm{E}+06$ & $1.65 \mathrm{E}+06$ & $8.52 E-06$ & $1.88 \mathrm{E}-01$ & $1.13 \mathrm{E}-03$ & $1.23 \mathrm{E}-09$ & 89.57 \\
\hline Hexadecane & $4.04 \mathrm{E}-03$ & $4.34 \mathrm{E}-02$ & $8.69 \mathrm{E}+05$ & $1.34 \mathrm{E}+06$ & $4.45 E-05$ & $7.75 \mathrm{E}-02$ & $5.58 \mathrm{E}-03$ & $2.21 \mathrm{E}-08$ & 89.56 \\
\hline Glycerol & $4.60 \mathrm{E}-06$ & $1.38 \mathrm{E}-06$ & $3.97 \mathrm{E}+07$ & $2.06 \mathrm{E}+06$ & $8.62 \mathrm{E}-04$ & 1.30 & $3.00 \mathrm{E}-05$ & $5.19 \mathrm{E}-09$ & 1.99 \\
\hline$\alpha$-Terpineol & $2.13 \mathrm{E}-04$ & $5.21 \mathrm{E}-04$ & $4.20 \mathrm{E}+06$ & $1.48 \mathrm{E}+06$ & $2.27 \mathrm{E}-04$ & $3.16 \mathrm{E}-01$ & $3.56 \mathrm{E}-04$ & $8.43 \mathrm{E}-09$ & 57.48 \\
\hline Polyethylene Glycol & $1.45 \mathrm{E}-01$ & 8.85 & $1.85 \mathrm{E}+05$ & $1.71 \mathrm{E}+06$ & $8.62 \mathrm{E}-04$ & $8.47 \mathrm{E}-03$ & $5.45 \mathrm{E}-01$ & $6.51 \mathrm{E}-05$ & 89.91 \\
\hline Canola Oil & $1.58 \mathrm{E}-02$ & $3.40 \mathrm{E}-01$ & $4.73 \mathrm{E}+05$ & $1.44 \mathrm{E}+06$ & $2.04 \mathrm{E}-03$ & $3.41 \mathrm{E}-02$ & $2.95 \mathrm{E}-02$ & $2.90 \mathrm{E}-06$ & 88.06 \\
\hline Castor Oil & $1.67 \mathrm{E}-02$ & $3.38 \mathrm{E}-01$ & $5.14 \mathrm{E}+05$ & $1.61 \mathrm{E}+06$ & $2.41 \mathrm{E}-02$ & $3.40 \mathrm{E}-02$ & $3.51 \mathrm{E}-02$ & $4.30 \mathrm{E}-06$ & 88.37 \\
\hline $\begin{array}{c}\text { 1-Ethyl-3- } \\
\text { methylimidazolium } \\
\text { tetrafluoroborate } \\
\text { ([EMIM][BF4]) }\end{array}$ & $5.29 \mathrm{E}-07$ & $6.28 \mathrm{E}-08$ & $1.01 \mathrm{E}+08$ & $1.78 \mathrm{E}+06$ & $6.07 E-09$ & 4.34 & $1.99 \mathrm{E}-06$ & $1.83 \mathrm{E}-15$ & 89.83 \\
\hline $\begin{array}{c}\text { Dielectric Oil } \\
\text { (Light Naphthenic } \\
\text { Petroleum Distillate) }\end{array}$ & $3.94 \mathrm{E}-02$ & 1.18 & $3.39 \mathrm{E}+05$ & $1.63 \mathrm{E}+06$ & $7.29 \mathrm{E}-04$ & $2.74 \mathrm{E}-02$ & $5.85 \mathrm{E}-02$ & $5.36 \mathrm{E}-06$ & 89.29 \\
\hline
\end{tabular}

Table 1. Characteristic properties for selected EHD liquid. Values in bold and italics denote the unfavorable property for jet coalescence.

The $d_{0}, t_{c}$ are the characteristic length for the jet and the local capillary time, as defined in Section 4.1. The local characteristic field strength $E_{0}$ is calculated by setting the local $B o_{E}$ to be 1 and the bulk field strength $E_{0 B}$ is calculated by using the bulk characteristic length $a$ for this relation assuming a volume of $\sim 5 \mu L . L_{0}, v_{z}, R_{0}$ are the characteristic jet length, end speed of the tip, front radius of a fully developed jet. $F_{k 0}$, and $\theta_{0}$ are the characteristic kinetic energy of the jet and half cone angle of the jet defined as: 


$$
\begin{gathered}
F_{k 0}=\pi \rho R_{0}^{2} L v_{z}^{2} \\
\theta_{0}=\operatorname{atan}\left(\frac{R_{0}}{L}\right)
\end{gathered}
$$

For the purposes of this table, $L$, the characteristic axial length, is based on a piecewise function:

$$
L= \begin{cases}L_{0}, & L_{0}<H_{0} \\ H_{0} \sim 1 \mathrm{~mm}, & L_{0} \geq H_{0}\end{cases}
$$

Since a jet length greater than the gap will lead to bridging and therefore will never form.

From Table 1, we can see different materials have different failure modes based on the geometry and electric field limit of the device ( mm gap size, electric field strength $\sim \mathrm{kV} / \mathrm{mm})$ :

(1) The characteristic times for the two types of silicone oil tested are too long thus their motion is not significant. In addition, the local field strength during the tip streaming process is well below the bulk field strength, meaning that field required for forming the jet is even smaller than the field required to move the bulk liquid. This oversupply of voltage/field might turn the tip streaming into a multi-jet electrospray mode ${ }^{31,32}$.

(2) For hexadecane and NMP, due to the short length of the fully developed jet, we would expect the jetting mode to be the main mode at a higher slenderness as the opposing droplets could not meet each other.

(3) Glycerol and PEG are able to form slender cone jet that is long enough to make a stable neck region between opposing droplets. As seen in Fig. 4, both liquids could form a stable bridge at a slenderness larger than 0.6. However, with the small cone angle of the jet, glycerol shows a $\mathrm{J}$ behavior at the slenderness where PEG shows a $\mathrm{BO}$ behavior.

(4) The cone angle of the jet for $\alpha$-Terpeniol is smaller than the critical value $\left(59.3^{\circ}\right)$ thus even if the jets could meet, they could not develop into a stable bridge. 


\section{CONCLUSIONS}

By placing $5 \mu \mathrm{L} \sim 15 \mu \mathrm{L}$ liquid material of different types in a parallel plate capacitor with a 1 $\mathrm{mm}$ thick dielectric layer, we have found five types of characteristic behavior modes of the liquid subjected to an axial direction DC electric field. Among them, in the bridging with debridging mode, the capillary bridge's existence could be fully controlled by the on and off of the electric field. By analyzing the free energy, we found for the materials that could achieve this behavior, the key point in achieving this state is to have a capillary bridge with higher surface energy than the droplets state and an electric field that makes the total free energy lower during the bridge state. Through specific combination of material properties, an electric field controlled switchable bridging and debridging mode has been found at intermediate gap size for liquid with several leaky dielectric materials. A small contact angle, the ability to form a tip streaming cone jet, and the coalescence of opposing jets are critical for achieving the electric field-controlled switching behavior. It is important to note that relatively few liquids are capable of undergoing this transition due to the need for multiple material properties to be within specific ranges. In particular, vegetable oils present the greatest opportunity as switching liquids since they are non-volatile, have wide switchable ranges, and are sustainable materials. This understanding of the materials requirements also provides the opportunity to formulate fluids that are co-optimized for stable bridging and some other properties, such as electrical or thermal conduction.

\section{ACKNOWLEDGMENT}

The authors want to thank US DOE for the funding of this work(DE-EE0008314). We also would like to thank Stepan Company for providing the NEOBEE 895. 


\section{REFERENCES}

1. Da Vinci, L., The notebooks of Leonardo da Vinci. Courier Corporation: 2012; Vol. 1. 2. $\quad$ Eggers, J.; Villermaux, E., Physics of liquid jets. Reports on Progress in Physics 2008, 71 (3), 036601.

3. Ramos, A.; González, H.; Castellanos, A., Experiments on dielectric liquid bridges subjected to axial electric fields. 1994, 6 (9), 3206-3208.

4. Saville, D. A., Stability of Electrically Charged Viscous Cylinders. Physics of Fluids 1971, 14 (6), 1095.

5. Sankaran, S.; Saville, D. A., Experiments on the stability of a liquid bridge in an axial electric field. Physics of Fluids A: Fluid Dynamics 1993, 5 (4), 1081-1083.

6. González, H.; McCluskey, F. M. J.; Castellanos, A.; Barrero, A., Stabilization of dielectric liquid bridges by electric fields in the absence of gravity. J. Fluid Mech. 1989, 206, 545-561.

7. Klingner, A.; Buehrle, J.; Mugele, F., Capillary Bridges in Electric Fields. Langmuir 2004, 20 (16), 6770-6777.

8. Klingner, A.; Herminghaus, S.; Mugele, F., Self-excited oscillatory dynamics of capillary bridges in electric fields. 2003, 82 (23), 4187.

9. Ramos, A.; Castellanos, A., Bifurcation diagrams of axisymmetric liquid bridges of arbitrary volume in electric and gravitational axial fields. 1993, 249 (-1), 207.

10. Petkov, P. V.; Radoev, B. P., Statics and dynamics of capillary bridges. Colloids and Surfaces A: Physicochemical and Engineering Aspects 2014, 460, 18-27.

11. Radoev, B.; Ivanov, I. T.; Petkov, P., Capillary bridge: Transition from equilibrium to hydrodynamic state. Colloids and Surfaces A: Physicochemical and Engineering Aspects 2016, 505, 98-105.

12. Mugele, F.; Baret, J.-C., Electrowetting: from basics to applications. Journal of Physics: Condensed Matter 2005, 17 (28), R705-R774.

13. Jian, G.; Gilhwan, C.; Ju, Y. S.; Chang-Jin, C. J. K. In Thermal switches based on coplanar EWOD for satellite thermal control, 2008 IEEE 21st International Conference on Micro Electro Mechanical Systems, 13-17 Jan. 2008; 2008; pp 848-851.

14. Cho, J.; Richards, C.; Bahr, D.; Jiao, J.; Richards, R., Evaluation of contacts for a MEMS thermal switch. Journal of Micromechanics and Microengineering 2008, 18 (10), 105012.

15. Cloupeau, M.; Prunet-Foch, B., Electrostatic spraying of liquids: Main functioning modes. Journal of Electrostatics 1990, 25 (2), 165-184.

16. Bird, J. C.; Ristenpart, W. D.; Belmonte, A.; Stone, H. A., Critical Angle for Electrically Driven Coalescence of Two Conical Droplets. Physical Review Letters 2009, 103 (16), 164502.

17. Ristenpart, W. D.; Bird, J. C.; Belmonte, A.; Dollar, F.; Stone, H. A., Non-coalescence of oppositely charged drops. Nature 2009, 461 (7262), 377-380.

18. Zeleny, J., Instability of Electrified Liquid Surfaces. Physical Review 1917, 10 (1), 1-6.

19. Gañán-Calvo, A. M.; López-Herrera, J. M.; Rebollo-Muñoz, N.; Montanero, J. M., The onset of electrospray: the universal scaling laws of the first ejection. Scientific Reports 2016, 6 (1), 32357. 
20. Gañán-Calvo, A. M.; Montanero, J. M., Revision of capillary cone-jet physics:

Electrospray and flow focusing. Phys Rev E 2009, 79 (6).

21. Collins, R. T.; Harris, M. T.; Basaran, O. A., Breakup of electrified jets. J. Fluid Mech. 2007, 588, 75-129.

22. Ferrera, C.; López-Herrera, J. M.; Herrada, M. A.; Montanero, J. M.; Acero, A. J., Dynamical behavior of electrified pendant drops. Physics of Fluids 2013, 25 (1), 012104.

23. Betelú, S. I.; Fontelos, M. A.; Kindelán, U.; Vantzos, O., Singularities on charged viscous droplets. Physics of Fluids 2006, 18 (5), 051706.

24. Collins, R. T.; Sambath, K.; Harris, M. T.; Basaran, O. A., Universal scaling laws for the disintegration of electrified drops. Proc Natl Acad Sci U S A 2013, 110 (13), 4905-4910.

25. Saville, D. A., Stability of Electrically Charged Viscous Cylinders. The Physics of Fluids 1971, 14 (6), 1095-1099.

26. Saville, D. A., Electrohydrodynamic Stability: Fluid Cylinders in Longitudinal Electric Fields. 1970, 13 (12), 2987.

27. Pawar, S. D.; Murugavel, P.; Lal, D. M., Effect of relative humidity and sea level pressure on electrical conductivity of air over Indian Ocean. Journal of Geophysical Research 2009, 114 (D2).

28. Ramos, A.; González, H.; Castellanos, A., Experiments on dielectric liquid bridges subjected to axial electric fields. Physics of Fluids 1994, 6 (9), 3206-3208.

29. Gañán-Calvo, A. M., Cone-Jet Analytical Extension of Taylor's Electrostatic Solution and the Asymptotic Universal Scaling Laws in Electrospraying. Physical Review Letters 1997, 79 (2), 217-220.

30. Collins, R. T.; Jones, J. J.; Harris, M. T.; Basaran, O. A., Electrohydrodynamic tip streaming and emission of charged drops from liquid cones. Nature Physics 2008, 4 (2), 149154.

31. Ryan, C. N.; Smith, K. L.; Stark, J. P. W., Characterization of multi-jet electrospray systems. Journal of Aerosol Science 2012, 51, 35-48.

32. Cloupeau, M.; Prunet-Foch, B., Electrostatic spraying of liquids in cone-jet mode.

Journal of Electrostatics 1989, 22 (2), 135-159. 